\title{
The link between chronic spontaneous urticaria and metabolic syndrome
}

Dermatology and Venereology Private Practice, Bari and Barletta, Italy

\section{KEY WORDS \\ cardiovascular risk; chronic urticaria; \\ chronic spontaneous urticaria; metabolic \\ syndrome; obesity}

\section{Corresponding author}

Gino Antonio Vena

Via Fanelli, 206/16 70125 Bari, Italy

Phone: +39080 5025794

E-mail: ginovena@gmail.com

\section{Doi}

10.23822/EurAnnACI.1764-1489.12

\begin{abstract}
Summary
Metabolic syndrome (MS) is a cluster of risk factors for cardiovascular disease and is considered a chronic low-level systemic inflammatory condition. Recent preliminary findings have shown an increased prevalence of MS among patients with chronic urticaria (CU) as compared to controls, with a particularly higher prevalence detected in patients with uncontrolled $C U$. Chronic spontaneous urticaria (CSU) appears to share some pathomechanisms with MS, including a pro-inflammatory state, increased oxidative stress, alterations in adipokine profile and activation of the coagulation system. Further studies are needed to assess the association of MS and its components with CU/CSU and to obtain more precise information regarding epidemiological aspects, clinical significance and implications. The aim of this review is to present the most relevant literature data on the link between CU/CSU and MS.
\end{abstract}

\section{Introduction}

\section{Chronic spontaneous urticaria}

Chronic urticaria $(\mathrm{CU})$ is characterized by recurrent wheals, angioedema, or both for at least six weeks. CU can be distinguished into two main groups depending on whether the lesions occur spontaneously [chronic spontaneous urticaria (CSU)] or are induced by specific physical-environmental stimuli [chronic inducible urticaria (CIndU)] (1).

Various aetiological factors and mechanisms have been implicated in the development of CSU, but a specific cause is unidentifiable in most cases. Disease pathogenesis remains incompletely understood, although an autoimmune basis is increasingly being recognized based on the presence of functional histamine-releasing autoantibodies in a subset of patients $(2,3)$. The autologous serum skin test (ASST) is a simple screening tool able to detect autore- activity related to the presence of circulating histamine-releasing factors of any type, and not only of functional autoantibodies (1). Current evidence supports the possible contribution of other pathomechanisms, including the dysregulation of intracellular signalling pathways in basophils and mast cells, an abnormal innate immunity response, and the simultaneous activation of inflammatory response and coagulation system (2-4). With regard to the last aspect in more detail, significantly increased circulating levels of inflammatory markers [such as C-reactive protein (CRP) and interleukin (IL)-6], fibrin degradation products and D-dimer have been associated with the active phase of CSU, in correlation with disease severity (5). The activation of coagulation in CSU is likely to take place through the involvement of eosinophils and tissue factor pathway with thrombin generation and increased vascular permeability (6). An elevated oxidative stress level was demonstrated in patients with CSU (7-9) in parallel with systemic inflammation (10). 
Nevertheless, the various mechanisms proposed so far individually cannot explain all the cases of CSU. CSU can be considered a heterogeneous multifactorial condition resulting from a complex interplay between the different pathogenic pathways and/or consisting of various subgroups driven by different pathomechanisms.

\section{Metabolic syndrome}

Metabolic syndrome (MS) is estimated to affect approximately $20-25 \%$ of the adult population and is a cluster of risk factors for cardiovascular disease, including atherogenic dyslipidaemia, glucose intolerance, arterial hypertension and central obesity. In particular, this syndrome has been found to cause a 5-fold increase in the risk of type 2 diabetes mellitus and 2-fold increase in the risk of cardiovascular disease over the next 5 to 10 years (11). Among the various definitions of MS, the most widely accepted and used are those developed by the World Health Organization (WHO), the European Group for the Study of Insulin Resistance (EGIR), the National Cholesterol Education Program-Third Adult Treatment Panel (NCEP-ATPIII), the American Association for Clinical Endocrinology (AACE) and the International Diabetes Federation (IDF) $(12,13)$. Insulin resistance is a required criterion for diagnosis in the WHO definition. According to the NCEP-ATP III 2001 definition, MS can be diagnosed in the presence of three or more of the following criteria: waist circumference $\geq 102 \mathrm{~cm}$ in men or $\geq 88 \mathrm{~cm}$ in women; triglyceride level $\geq 150 \mathrm{mg} / \mathrm{dl}$; high-density lipoprotein (HDL) cholesterol level $<40 \mathrm{mg} / \mathrm{dl}$ in men or $<50 \mathrm{mg} / \mathrm{dl}$ in women; blood pressure $\geq 130 / 85 \mathrm{mmHg}$; and fasting plasma glucose level $\geq 100 \mathrm{mg} / \mathrm{dl}$ (14).

In addition, the syndrome encompasses a systemic prothrombotic and pro-inflammatory state as relevant features with endothelial dysfunction and hypercoagulability (12), and can be considered a chronic low-level systemic inflammatory condition. Elevated circulating cytokines and acute-phase reactants, and abnormalities in clotting and antifibrinolytic factors have been found, such as high levels of IL-1, IL-6, tumor necrosis factor (TNF)-alpha, CRP, fibrinogen, tissue factor, factor VII, and plasminogen activator inhibitor (PAI)-1. Adipose tissue plays a crucial role in conditioning the prothrombotic risk, as well as systemic inflammation, through an altered secretion of adipokines that enhance and sustain the inflammatory response and the hepatic production of CRP $(12,15)$.

The role of oxidative stress in MS components, as well as in the onset of MS-related cardiovascular complications, has been remarked (16). A linear increase in the concentration of the thrombotic marker D-dimer was detected in MS, in correlation with chronic inflammation, oxidative stress and altered hemorheology (17).

\section{Methods}

Considering the above-mentioned premises outlining possible shared inflammatory pathways between CSU/CU and MS, we reviewed the available literature on the association and interaction between such conditions. PubMed searches were conducted using the keywords "chronic urticaria" or "chronic spontaneous urticaria", and "metabolic syndrome" and single MS components. The search was also extended to cover other relevant topics, such as "adipokines" and "cardiovascular risk". Articles concerning heterogeneous patient populations without proper selection of CU or CSU patients (e.g. subjects with unspecified urticaria or dermatitis / urticaria) were excluded.

\section{Association with MS or MS components}

The link between CU and MS has been highlighted by a cross-sectional study carried out on 131 Korean patients with CU (18). MS, diagnosed using the NCEP-ATP III definition, was present in $29.8 \%$ of patients and $17.8 \%$ of subjects in the control group, giving rise to a statistically significant difference. Comparison of each component of MS between patients and controls showed a statistically higher frequency of central obesity, hypertriglyceridemia and hyperglicemia in CU patients. Compared with CU patients without MS, those with MS were found to be significantly older and more often males, to have more frequent negative ASST results, less frequent angioedema and higher urticaria activity score. Therefore, the presence of MS was more strictly associated with nonautoreactive form and correlated with disease severity but not with angioedema. Logistic regression analysis revealed that the presence of MS was an independent predictor of uncontrolled $\mathrm{CU}$ defined by the absence of symptom control after 3 months of treatment. Moreover, patients with CU and MS had higher serum levels of eosinophil cationic protein, TNF-alpha, and complement factors $\mathrm{C} 3$ and $\mathrm{C} 4$.

An investigation on multiple epidemiological features of CSU was conducted using the Health Search IMS Longitudinal Patient Database (HSD), that contains electronic medical records of patients aged $\geq 15$ years with at least 1 year of medical history registered by 700 selected Italian general practitioners (19). In this population-based study, the risk of CSU was shown to be significantly increased (adjusted hazard ratio, 1.40; 95\% confidence interval, 1.17-1.67) in the presence of obesity (ICD-9CM code 278.0 or body mass index $\geq 30$ ).

Rogala et al. (20) described the association between impaired glucose tolerance and recurrent angioedema without wheals. The authors noted that fasting plasma glucose levels, random blood glucose levels and oral glucose tolerance testing values were significantly higher in patients with angioedema alone as compared to CSU patients. 
A relationship between $\mathrm{CU}$ and serum lipids and fatty acids was previously suggested by Kobayashi (21), who hypothesized the role of omega- 6 and omega- 3 series of polyunsaturated fatty acids and lipid peroxidation as mediators in CU.

The association between hyperlipidemia and CU has recently been evaluated by a case-control study using a population-based dataset in Taiwan, and involving 9,798 adults with CU and 9,798 sex- and age-matched controls (22). These subjects were examined for whether they had received a prior diagnosis of hyperlipidemia (pure hypercholesterolemia; pure hypertriglyceridemia; mixed hyperlipidemia; hyperchylomicronemia; other and unspecified hyperlipidemia). Compared to patients without CU, CU patients independently had a 1.65-fold ( $95 \%$ confidence interval, 1.55-1.76; $\mathrm{p}<0.001$ ) increased risk of having a prior diagnosis of hyperlipidemia, after adjusting for relevant covariates. The analysis of patients with atopic dermatitis matched by sex, age group and index year disclosed that atopic dermatitis was not associated with a previous hyperlipidemia diagnosis, ruling out a relationship of this diagnosis with inflammatory skin diseases in general.

A prospective study in a cohort of 228 consecutive adults with moderate to severe CSU revealed that blood hypertension was associated with extended duration of CSU and influenced disease remission (23). In particular, persistence of CSU after 5 years was still detected in $74 \%$ and $54 \%$ of CSU patients with and without hypertension, respectively.

The association between CSU and hypertension was examined by a retrospective cohort study of 2,460 patients with CSU and 9,840 age-, sex-, and index year-matched control patients, using the National Health Insurance of Taiwan database (24). The median follow-up periods were 7.13 years and 7.20 years for the CSU cohort and for the control group, respectively. Patients with CSU were found to have a 1.37 -fold (95\% confidence interval, 1.22-1.53) greater risk of developing subsequent hypertension than the non-CSU cohort after adjusting for sex, age, comorbidities, and nonsedating antihistamine use.

\section{Role of adipokines}

The association between CSU and MS as a whole or individual components is very interesting, and might support the existence of shared factors and/or mediators. Systemic inflammation is a phenomenon common for MS and CSU acting as a possible link between the two conditions.

In this context, the role of obesity appears to be particularly relevant.

In recent years, visceral adipose tissue, previously thought to be an inert tissue, has been shown to be an active secretory organ and a source of adipokines crucially involved in immunity and inflammation $(25,26)$. As a consequence, a connection between obesity and autoimmunity has been hypothesized and appears to be corroborated by several findings. Obesity may in fact contribute to the onset and progression of various autoimmune conditions. A systematic review disclosed that obesity significantly increases the risk of rheumatoid arthritis, multiple sclerosis, psoriasis and psoriatic arthritis, and worsens the course of rheumatoid arthritis, systemic lupus erythematosus, inflammatory bowel disease, psoriasis and psoriatic arthritis (26).

Adipokines have been linked to the pathogenesis of MS and its comorbidities through their effects on vascular function and inflammation. Human mast cells may be also a direct target of adipokine activity $(27,28)$.

Systemic inflammation generated during obesity is characterized by an oversecretion of inflammatory markers such as CRP, IL- 6, IL-1beta, TNF-alpha, leptin and visfatin, with a simultaneous hyposecretion of anti-inflammatory and anti-atherogenic substances $(12,15,29)$.

Trinh et al. recently described an imbalance in pro- and anti-inflammatory adipokines in CU. They assessed serum levels of adiponectin, leptin, lipocalin-2 (LCN2), IL-10, IL-6, and TNF-alpha in $191 \mathrm{CU}$ patients and 89 healthy controls (30). In $\mathrm{CU}$ patients compared to controls, mean levels of serum LCN2, TNF-alpha, IL-6, and IL-10 were significantly higher, and adiponectin levels were significantly lower.

Adiponectin is one of the most abundant peptide hormones derived from adipose tissue and has anti-inflammatory and anti-oxidative properties. Adiponectin acts as a key regulator of innate immune system and progression of inflammation and metabolic disorders. This protein downregulates the expression and release of a number of pro-inflammatory immune mediators, plays a major role in glucose and lipid metabolism and prevents development of vascular changes. Hypoadiponectinemia is associated with a prothrombotic state, atherosclerosis, obesity, and MS (31-33).

LCN2, also known as neutrophil gelatinase-associated lipocalin (NGAL), is a secreted glycoprotein that belongs to the lipocalin family of proteins that transport small hydrophobic ligands, such as steroid hormones, lipids and retinoids, and has recently been characterized as a member of the adipokines superfamily (34). This adipokine plays a role in innate immunity and acute phase response to infection, and has been reported to have roles in the induction of apoptosis in hematopoietic cells, modulation of oxidative stress and inflammation, and metabolic homeostasis. LCN2 has been investigated as a diagnostic and prognostic biomarker in numerous pathological conditions, such as cancer, tissue injury, inflammation and autoimmunity $(34,35)$. Recently, LCN2 has also been proposed as a possible biomarker for disease activity and clinical response to antihistamine therapy in CU. In particular, in the study performed by Trinh et al. (30), elevated levels of LCN2 in CU patients were inversely correlated with urticarial activity score, while LCN2 was significantly increased in patients with responsive $\mathrm{CU}$ compared to those with $\mathrm{CU}$ resistant to antihistamines. 


\section{Assessment of cardiovascular risk}

Several immune-mediated inflammatory disorders, including rheumatoid arthritis, systemic lupus erythematosus, psoriatic disease and intestinal bowel diseases, have been associated with an elevated cardiovascular burden (25,36-39). The pathophysiological basis of such an increased risk is not completely understood, but MS and obesity in particular, with a dysregulated secretion of pro-inflammatory adipokines, could be major contributing features. Low-grade inflammation, that occurs in both $\mathrm{CSU}$ and MS, plays an important role in atherosclerosis.

In a nationwide Danish cohort using prospectively collected administrative data, the assessment of cardiovascular risk was performed in 2,215 patients with CU and 977 with CIndU (40). Patients were adults who received a first time diagnosis between 1997 and 2012, and were matched with healthy controls according to a 1:30 ratio. After adjustment for potential confounding factors, there were not significantly higher risks of myocardial infarction, ischaemic stroke, cardiovascular death, or major adverse cardiovascular events in the total population of patients with CU compared to controls. Similarly, no increased risk in any of the above-mentioned cardiovascular outcomes was seen in patients with CIndU, as well as in those with CSU.

Moreover, in patients with long-lasting moderate-severe CSU compared to healthy subjects, Grzanka et al. did not find any increase of circulating levels of matrix GLA protein, a biomarker of arterial calcification that is known to be overexpressed in patients with atherosclerosis (41).

\section{Conclusions}

Preliminary evidence from the limited data currently available seems to support the association between CU/CSU and MS. The presence of MS was shown to be associated with nonautoreactive forms and to act as an independent predictor of severe and uncontrolled disease, suggesting the possible involvement of the low-grade inflammatory status related to MS in perpetuating and exacerbating the inflammatory processes underlying CSU pathogenesis. CSU appears to share some pathobiological pathways with MS, including a pro-inflammatory state, increased oxidative stress, alterations in adipokine profile and activation of the coagulation system.

The role of obesity appears to be of particular interest. It has been reported that the serum levels of mast cell-derived tryptase and the number of adipose tissue mast cells are increased in obese patients and high fat diet-fed mice, respectively $(42,43)$. Mast cells in adipose tissue have been proposed to contribute to the pathophysiology of obesity and diabetes and related systemic inflammation (44).

MS and some components in particular (e.g. obesity) might also affect the response to the pharmacotherapy of CSU. Further research is needed to assess the association with MS and the practical implications in terms of prognosis and treatment response among CSU patients. The influence of drugs used to manage MS components on CSU severity and course should also be defined.

While CU has been associated with MS components in recent reports, there appears to be no increased prevalence of cardiovascular disease among $\mathrm{CU}$ patients, possibly because of the relatively short disease duration, that is unable to confer a relevant arteriosclerotic risk following sustained low-level inflammation, unlike other chronic long-standing skin conditions, such as psoriasis (40).

\section{Conflict of interest}

During the last three years, G.A. Vena has been a speaker and/ or an advisory board member for Novartis Farma and Pfizer, and N. Cassano has been a scientific consultant for Leo Pharma, Novartis Farma, and Pfizer.

\section{References}

1. Zuberbier T, Aberer W, Asero R, Bindslev-Jensen C, Brzoza Z, Canonica GW et al. The EAACI/GAALEN/EDF/WAO Guideline for the definition, classification, diagnosis, and management of urticaria: the 2013 revision and update. Allergy. 2014;69(7):868-87.

2. Najib U, Sheikh J. The spectrum of chronic urticaria. Allergy Asthma Proc. 2009;30(1):1-10.

3. Altman K, Chang C. Pathogenic intracellular and autoimmune mechanisms in urticaria and angioedema. Clin Rev Allergy Immunol. 2013;45(1):47-62.

4. Jain S. Pathogenesis of chronic urticaria: an overview. Dermatol Res Pract. 2014;2014:674709.

5. Kolkhir P, Andre F, Church MK, Maurer M, Metz M. Potential blood biomarkers in chronic spontaneous urticaria. Clin Exp Allergy. 2017;47(1):19-36.

6. Cugno M, Asero R, Tedeschi A, Lazzari R, Marzano AV. Inflammation and coagulation in urticaria and angioedema. Curr Vasc Pharmacol. 2012;10(5):653-8.

7. Raho G, Cassano N, D’Argento V, Vena GA, Zanotti F. Over-expression of $\mathrm{Mn}$-superoxide dismutase as a marker of oxidative stress in lesional skin of chronic idiopathic urticaria. Clin Exp Dermatol. 2003;28(3):318-20.

8. Cassano N, Raho G, Filieri M, D’Argento V, Amoruso A, Filotico $\mathrm{R}$, Vena GA. Influence of desloratadine on oxidative stress markers in patients with chronic idiopathic urticaria. Int J Dermatol. 2006;45(4):394-6.

9. Akbas A, Kilinc F, Sener S, Aktaş A, Baran P, Ergin M. Investigation of thiol-disulphide balance in patients with acute urticaria and chronic spontaneous urticaria. Cutan Ocul Toxicol. 2017;36(3):205-10.

10. Rajappa M, Chandrashekar L, Sundar I, Munisamy M, Ananthanarayanan PH, Thappa DM, Toi PCh. Platelet oxidative stress and systemic inflammation in chronic spontaneous urticaria. Clin Chem Lab Med. 2013;51(9):1789-94.

11. Alberti KG, Eckel RH, Grundy SM, Zimmet PZ, Cleeman JI, Donato $\mathrm{KA}$, et al. Harmonizing the metabolic syndrome: a joint inter- 
im statement of the International Diabetes Federation Task Force on Epidemiology and Prevention; National Heart, Lung, and Blood Institute; American Heart Association; World Heart Federation; International Atherosclerosis Society; and International Association for the Study of Obesity. Circulation. 2009;120(16):1640-5.

12. Kaur J. A comprehensive review on metabolic syndrome. Cardiol Res Pract. 2014;2014:943162.

13. O’Neill S, O’Driscoll L. Metabolic syndrome: a closer look at the growing epidemic and its associated pathologies. Obes Rev. 2015;16(1):1-12.

14. Expert panel on detection, evaluation and treatment of high blood cholesterol in adults (Adult Treatment Panel III): Executive summary of the third report of the national cholesterol education program (NCEP) JAMA 2001;285(19):2486-97.

15. Matsuzawa Y. The metabolic syndrome and adipocytokines. Expert Rev Clin Immunol. 2007;3(1):39-46.

16. Bonomini F, Rodella LF, Rezzani R. Metabolic syndrome, aging and involvement of oxidative stress. Aging Dis. 2015;6(2):109-20.

17. Gyawali P, Richards RS. Association of altered hemorheology with oxidative stress and inflammation in metabolic syndrome. Redox Rep. 2015;20(3):139-44.

18. Ye Y-M, Jin H-J, Hwang E-K et al. Co-existence of chronic urticaria and metabolic syndrome: Clinical implications. Acta Derm Venereol. 2012; 93(2):156-60.

19. Lapi F, Cassano N, Pegoraro V, Cataldo N, Heiman F, Cricelli I, Levi M, Colombo D, Zagni E, Cricelli C, Vena GA. Epidemiology of chronic spontaneous urticaria: results from a nationwide, population-based study in Italy. Br J Dermatol. 2016;174(5):996-1004.

20. Rogala B, Bozek A, Glück J, Rymarczyk B, Jarzab J, Maurer M. Coexistence of angioedema alone with impaired glucose tolerance. Int Arch Allergy Immunol. 2014;165(4):265-9.

21. Kobayashi S. Investigation of the roles of the substances in serum lipids and their constitutive fatty acids in chronic urticaria. J Dermatol. 1989;16(3):196-206.

22. Chung SD, Wang KH, Tsai MC, Lin HC, Chen CH. Hyperlipidemia is associated with chronic urticaria: A population-based study. PLoS One 2016;11(3):e0150304.

23. Nebiolo F, Bergia R, Bommarito L, Bugiani M, Heffler E, Carosso A, Castiglioni G, Guida G, Badiu I, Pizzimenti S, Mietta S, Ferrero N, Rolla G. Effect of arterial hypertension on chronic urticaria duration. Ann Allergy Asthma Immunol. 2009;103(5):407-10.

24. Chang HW, Cheng HM, Yen HR, Hsu CY, Lee YC, Chiang JH, Sun MF. Association between chronic idiopathic urticaria and hypertension: A population-based retrospective cohort study. Ann Allergy Asthma Immunol. 2016;116(6):554-8.

25. Abella V, Scotece M, Conde J, López V, Lazzaro V, Pino J, Gómez-Reino JJ, Gualillo O. Adipokines, metabolic syndrome and rheumatic diseases. J Immunol Res. 2014;2014:343746.

26. Versini M, Jeandel PY, Rosenthal E, Shoenfeld Y. Obesity in autoimmune diseases: Not a passive bystander. Autoimmun Rev. 2014;13(9):981-1000.

27. Sismanopoulos N, Delivanis DA, Mavrommati D, Hatziagelaki E, Conti P, Theoharides TC. Do mast cells link obesity and asthma? Allergy. 2013;68(1):8-15.
28. Taildeman J, Pérez-Novo CA, Rottiers I, Ferdinande L, Waeytens A, De Colvenaer V, Bachert C, Demetter P, Waelput W, Braet K, Cuvelier CA. Human mast cells express leptin and leptin receptors. Histochem Cell Biol. 2009;131(6):703-11.

29. Debnath M, Agrawal S, Agrawal A, Dubey GP. Metaflammatory responses during obesity: Pathomechanism and treatment. Obes Res Clin Pract. 2016;10(2):103-13.

30. Trinh HK, Pham DL, Ban GY, Lee HY, Park HS, Ye YM. Altered systemic adipokines in patients with chronic urticaria. Int Arch Allergy Immunol. 2016;171(2):102-10.

31. Fisman EZ, Tenenbaum A. Adiponectin: a manifold therapeutic target for metabolic syndrome, diabetes, and coronary disease? Cardiovasc Diabetol. 2014;13:103.

32. Esfahani M, Movahedian A, Baranchi M, Goodarzi MT. Adiponectin: an adipokine with protective features against metabolic syndrome. Iran J Basic Med Sci. 2015;18(5):430-42.

33. Luo Y, Liu M. Adiponectin: a versatile player of innate immunity. J Mol Cell Biol. 2016;8(2):120-8.

34. Abella V, Scotece M, Conde J, Gómez R, Lois A, Pino J, Gómez-Reino JJ, Lago F, Mobasheri A, Gualillo O. The potential of lipocalin-2/NGAL as biomarker for inflammatory and metabolic diseases. Biomarkers. 2015;20(8):565-71.

35. Chakraborty S, Kaur S, Guha S, Batra SK. The multifaceted roles of neutrophil gelatinase associated lipocalin (NGAL) in inflammation and cancer. Biochim Biophys Acta. 2012;1826(1):129-69.

36. Vena GA, Vestita M, Cassano N. Psoriasis and cardiovascular disease. Dermatol Ther. 2010;23(2):144-51.

37. Frieder J, Ryan C. Psoriasis and cardiovascular disorders. G Ital Dermatol Venereol. 2016;151(6):678-93.

38. Husni ME. Comorbidities in psoriatic arthritis. Rheum Dis Clin North Am. 2015;41(4):677-98.

39. Michalak A, Mosińska P, Fichna J. Common links between metabolic syndrome and inflammatory bowel disease: Current overview and future perspectives. Pharmacol Rep. 2016;68(4):837-46.

40. Egeberg A, Kofoed K, Gislason GH, Vestergaard C, Thyssen JP. Cardiovascular risk is not increased in patients with chronic urticaria: A retrospective population-based cohort study. Acta Derm Venereol. 2017;97(2):261-2.

41. Grzanka A, Machura E, Misiolek M, Polaniak R, Kasperski J, Kasperska-Zajac A. Systemic inflammatory response and calcification markers in patients with long lasting moderate-severe chronic spontaneous urticaria. Eur J Dermatol. 2015;25(1):26-8.

42. Liu J, Divoux A, Sun J, Zhang J, Clément K, Glickman JN, Sukhova GK, Wolters PJ, Du J, Gorgun CZ, Doria A, Libby P, Blumberg RS, Kahn BB, Hotamisligil GS, Shi GP. Genetic deficiency and pharmacological stabilization of mast cells reduce diet-induced obesity and diabetes in mice. Nat Med. 2009;15(8):940-5.

43. Moreno M, Puig J, Serrano M, Moreno-Navarrete JM, Ortega F, Ricart W, Fernandez-Real JM. Circulating tryptase as a marker for subclinical atherosclerosis in obese subjects. PLoS One. 2014;9(5):e0097014.

44. Divoux A, Moutel S, Poitou C, Lacasa D, Veyrie N, Aissat A, Arock M, Guerre-Millo M, Clément K. Mast cells in human adipose tissue: link with morbid obesity, inflammatory status, and diabetes. J Clin Endocrinol Metab. 2012;97(9):E1677-85. 